\title{
Le logement social à Oran. Conception, usages et ébauche d'évaluation
}

How to estimate the social housing after half a century of construction in Oran? Gestaltung, Nutzung und Auswertung der sozialen Wohnungen in Oran

\section{Najet Mouaziz-Bouchentouf}

\section{OpenEdition}

\section{Journals}

Electronic version

URL: http://journals.openedition.org/rge/5312

DOI: $10.4000 /$ rge.5312

ISSN: 2108-6478

\section{Publisher}

Association des géographes de l'Est

\section{Printed version}

Date of publication: 30 December 2014

ISSN: 0035-3213

\section{Electronic reference}

Najet Mouaziz-Bouchentouf, «Le logement social à Oran. Conception, usages et ébauche d'évaluation », Revue Géographique de l'Est [Online], vol. 54 / n³-4 | 2014, Online since 11 February 2015, connection on 08 September 2020. URL : http://journals.openedition.org/rge/5312 ; DOI https://doi.org/10.4000/rge.5312

This text was automatically generated on 8 September 2020

Tous droits réservés 


\title{
Le logement social à Oran. Conception, usages et ébauche d'évaluation
}

\author{
How to estimate the social housing after half a century of construction in Oran? \\ Gestaltung, Nutzung und Auswertung der sozialen Wohnungen in Oran
}

Najet Mouaziz-Bouchentouf

1 En cinquante ans d'indépendance, les efforts de l'Algérie concernant le logement sont appréciables même si ce qui est communément appelé la crise de logement n'est pas entièrement éradiqué. Depuis les années 1970, date des premiers programmes de logements sociaux, les praticiens dessinent et réalisent des logements que les chercheurs étudient, travaillant chacun de son côté en se tournant le dos. Autant les sociologues, les architectes que les géographes ont analysé le logement sous tous ses aspects : "L'espace résidentiel a été retenu comme objet de recherche pour ce qu'il renseigne sur les pratiques habitantes » (Lakjaa, 1998). Le logement collectif se prête à l'étude du fait de la position « exogène » de l'habitant qui « est exclu dès la conception de la production de son logement » (Arrif, 2000). Les professionnels quant à eux pris dans l'étau de l'urgence, des surfaces réduites au minimum et des prix plafonnés, reproduisent les mêmes formes et dispositions depuis les années 1970 dans une sorte d'autisme envers les résultats des recherches universitaires d'une part et l'évolution de la famille algérienne et de son mode de vie d'autre part. Cette indifférence au «sort» des logements se lit également à travers le désintérêt des maîtres d'ouvrage ${ }^{1} \mathrm{du}$ fait de l'absence d'études d'évaluation de leurs produits. Le propos ici est de jeter des passerelles entre la recherche et la profession, trois questions majeures émergent : comment la famille algérienne reconfigure le logement conçu sans elle? Quelles dispositions du logement sont bien reçues et quelles autres sont rejetées? Les conditions de réalisation du logement social favorisent-elles une attention vis-à-vis de l'usage des habitants ?

2 S'interroger sur la réception par les habitants de leurs logements est un thème récurrent depuis Pessac de Le Corbusier (Boudon, 1977) et Les pavillonnaires (Davidovitch, 1968). En France, l'échec des grands ensembles fait qu'on ne se méfie plus de la parole 
des habitants ${ }^{2}$ (Léger, Decup-Pannier, 2005) comme en témoigne la préoccupation du Plan Construction relative à la qualité d'usage des ensembles de logements et le classement $\mathrm{REX}^{3}$ (Tric, 1999). Au Maghreb, la confrontation du plan et sa transformation met au jour les compétences et les aptitudes des habitants de catégories sociales diverses à reformuler leurs logements (Pinson, 1994; Arrif, 2000 ; Bekkar, 2000 ; Belguidoum, Millet, 1985).

Cet article présente les premiers résultats d'une recherche sur le logement social à Oran qui, à l'instar des villes algériennes, a reçu depuis l'indépendance sa part de logements sociaux. Jusqu'à l'année $2000,81 \%$ du parc logement est social avec $48 \%$ en collectif et $42,6 \%$ en individuel (Said, 2005). Il est le fruit d'un travail de relevés de logements et d'entretiens avec les habitants qui confrontent le logement livré à celui habité. Il s'agit de rendre compte des compétences des habitants à remodeler l'espace, à le rendre conforme à leurs usages et à le personnaliser, le but étant d'atteindre une meilleure habitabilité. L'intérêt a porté sur la ZHUN ${ }^{4}$ Maraval (1980) et l'ensemble des 365 Logements Hai-Essabah (2000) qui sont livrés à vingt ans d'intervalle, une temporalité suffisante pour étudier l'évolution du logement social. L'exhaustivité n'étant pas un objectif premier, cette enquête préliminaire ne cible pas un échantillon représentatif de chaque quartier, la ZHUN Maraval dont la réalisation s'est étalée sur presque vingt ans ne comprend pas moins de 3000 logements. L'observation et l'entretien semi-directif ${ }^{5}$ avec les habitants sont les techniques utilisées lors de cette étude, les logements visités n'ont pas été véritablement choisis mais des occasions saisies quand leurs habitants ont bien voulu se soumettre aux questions et dévoiler leurs façons de vivre et leurs souhaits et désirs envers leur logement. Après un rappel des modèles hérités de la colonisation et de la typologie adoptée pour le logement social au lendemain de l'indépendance, suivra un exposé des usages de ces logements à travers les travaux d'autres auteurs et les résultats de l'enquête sur Oran complétés par le point de vue des architectes et des maîtres d'ouvrage.

\section{Aux sources du logement social : les modèles d'avant l'indépendance}

La question de l'habitat pour les musulmans est posée par l'administration française dès les années 1930, à l'occasion du centenaire de la colonisation. On retiendra la volonté de réfléchir à un modèle hybride, une architecture qui ouvre la voie vers l'assimilation progressive des familles algériennes et leur adoption des habitudes européennes, tout en veillant à respecter leurs vieilles coutumes (Celik, 1997). De cette période, le pays hérite d'études théoriques ${ }^{6}$ ou de projets réalisés.

5 C'est en 1935 que François Bienvenu réalise la première cité indigène au boulevard Verdun qui jouxte la Casbah d'Alger (figure 1a). C'est une combinaison de deux modèles, le traditionnel avec l'organisation des logements autour d'un patio, et le moderne arborant la verticalité et offrant les commodités des habitations destinées aux logements des européens. Le constat de ne pas pouvoir reconstruire à l'identique les maisons traditionnelles n'empêche en rien qu'elles fascinent tous ceux qui les découvrent. En 1936, Marcel Lathuillière (cité par Picard, 1994) déclare que «Les architectes d'Algérie ont combattu la routine et le pastiche et découvert une esthétique qui s'adapte aux exigences de la construction et de la vie moderne en même temps qu'aux nécessités naturelles commandées par le climat et par le site", et Le Corbusier (1935) de dire "Les 
civilisés vivent comme les rats dans des trous [...]. Les barbares vivent dans la quiétude, le bienêtre ». Ces préoccupations se prolongent jusqu'aux années 1950 lors de la construction des cités de recasement ou de transit pour résorber les bidonvilles qui se sont formés aux abords des principales villes algériennes. Il en est ainsi pour la cité Djenane el Hassan (1956-1958) œuvre de Roland Simounet (figure 1c) disciple de Le Corbusier mais aussi très imprégné de l'architecture locale. Cet ensemble de logements qui se distingue par sa ressemblance avec le projet Rob et Roq à Cap-Martin de Le Corbusier (Almi, 2002) est une belle symbiose entre le moderne et le traditionnel. À Oran, le député-maire Henri Fouques-Duparc fait construire la cité musulmane du Petit Lac (1954-1959), un habitat horizontal offrant à la famille musulmane les commodités modernes et l'intimité d'une cour autour de laquelle s'organisent les espaces de vie (figure 1d). En 1953, le maire d'Alger Jacques Chevallier fait construire par Fernand Pouillon trois ensembles de logements sociaux dont une partie est réservée aux musulmans. Au-delà des références aux fortifications turques, aux rues de la Casbah et au sud algérien, c'est une réelle volonté de donner une dignité et une grandeur comparables à celles du centre-ville européen à ces logements sociaux suburbains (Deluz, 1988).

Les logements sociaux d'avant l'indépendance :

Figure 1a : Projet théorique de cité indigène, 1936, Louis Bérthy

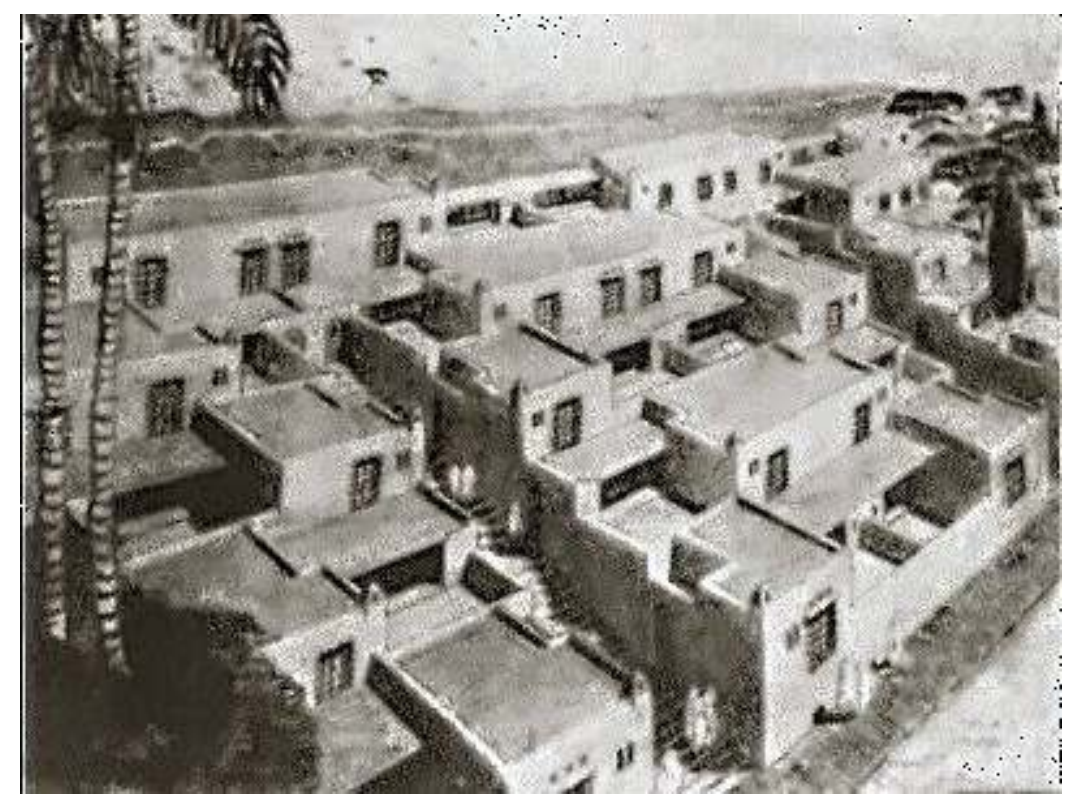


Figure 1b : Cité indigène du boulevard de Verdun, Alger, 1935, François Bienvenu

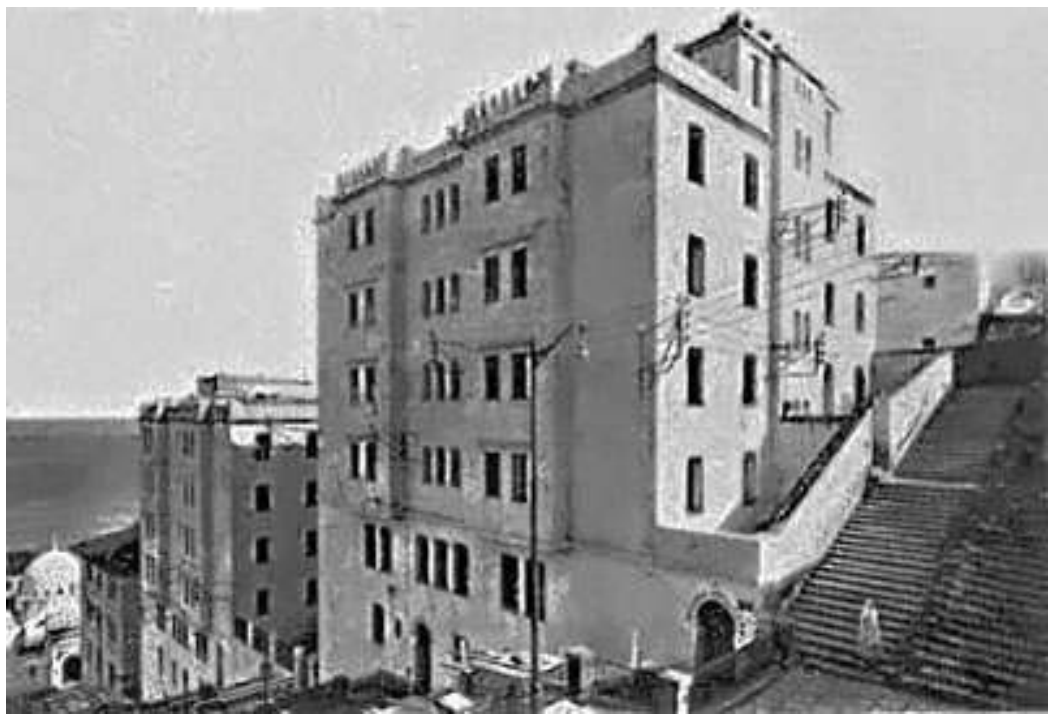

Figure 1c : Djenan-El-Hassan à Alger, 1956-1958, Roland Simounet

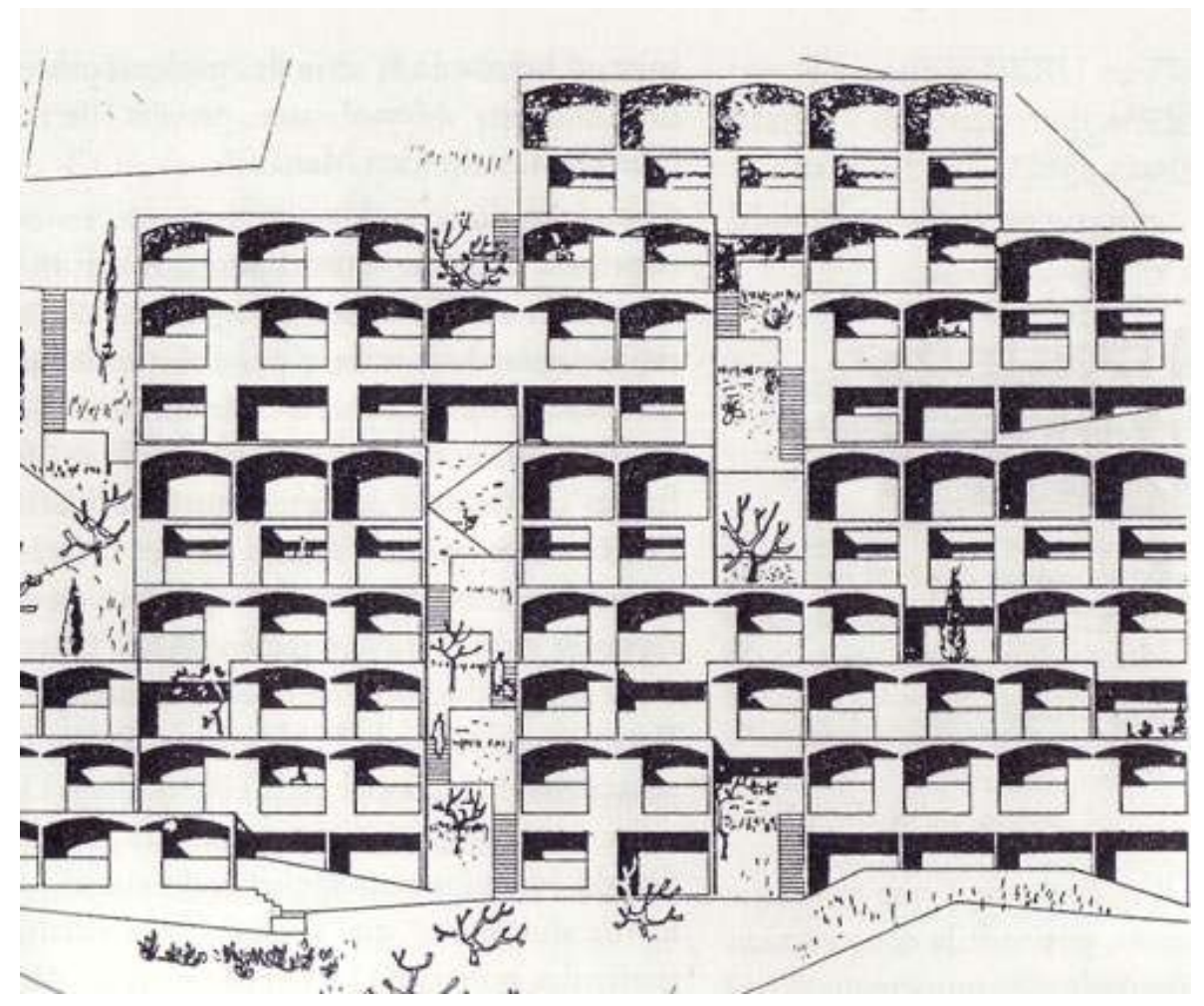


Figure 1d : Cité musulmane du Petit Lac à Oran (1954-1959)

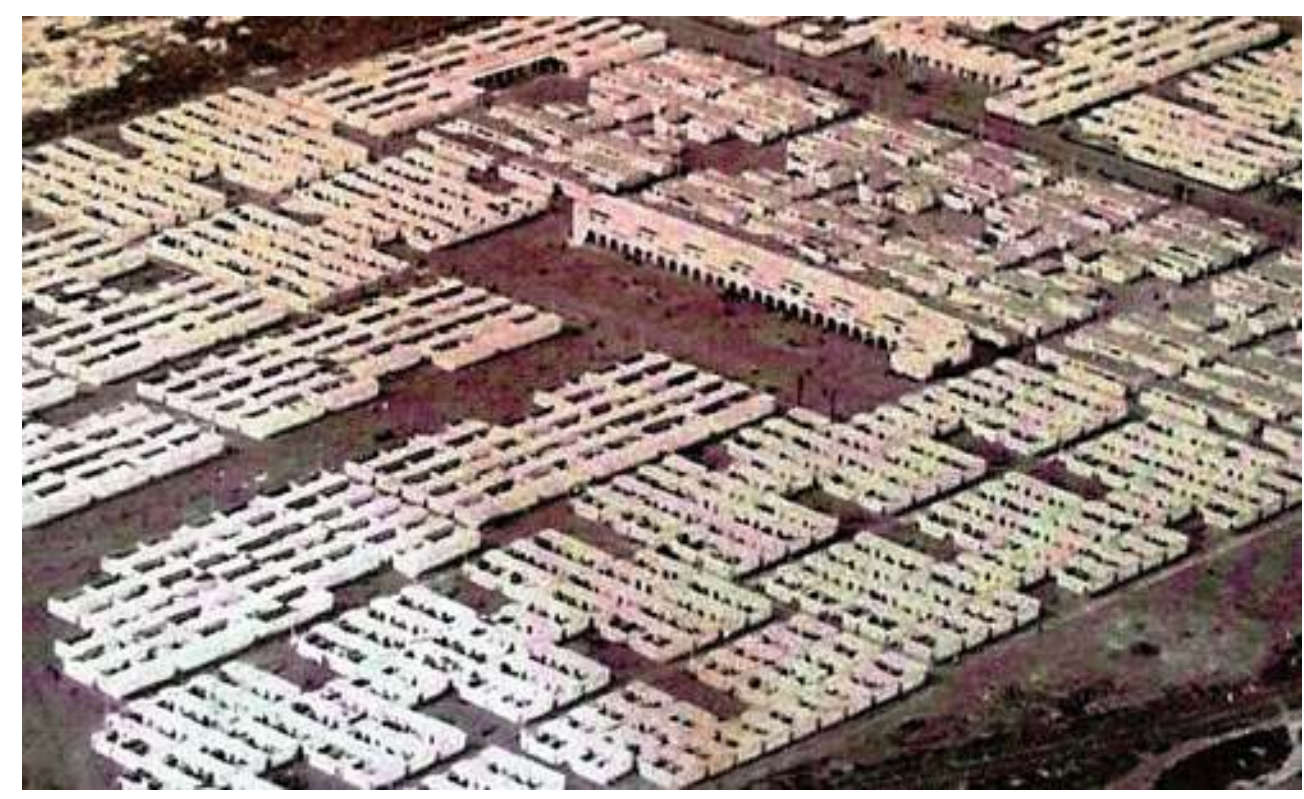

6 Ces interrogations sur le modèle d'habitat à adopter disparaîtront avec le plan de Constantine. À ce titre, Jean-Jacques Deluz (1988) résume bien l'évolution du logement en Algérie depuis la conquête française: "Pour la masse moyenne des architectes, la clientèle de la grosse bourgeoisie coloniale voulait du "néo-classique", le promoteur de l'aprèsguerre veut du "moderne", l'équipe de Chevallier demandera du "caractère", les technocrates du plan de Constantine $d u$ "technique" et après l'indépendance le nouveau régime exigera du "social". ». Bien que ces discussions se soient prolongées jusqu'à la fin de la domination française, leur intensité diminue, et les aspects socioculturels ont cédé la place à des préoccupations quantitatives et techniques. Les mots d'ordre du plan de Constantine porté et exécuté par les ingénieurs des Ponts et Chaussées et les polytechniciens sont rapidité, efficacité et quantité. Ils laissent peu de place au côté romantique de l'urbanisme et de l'architecture accusé de les retarder dans leur mission (Deluz, 2010). Il y a aussi l'intime conviction que les familles musulmanes aspirent à ce logement vertical de type européen avec ses commodités et ses vues directes même s'il est réduit à son expression la plus simple, et qu'au contraire elles rejettent le logement traditionnel qui par la cour n'ouvre la vue que vers le ciel (Meyer, 1958).

7 À son indépendance, l'Algérie hérite d'un parc de logements urbain appréciable dont une partie est libérée par les européens suite à leur exode. Des logements « différents ${ }^{7}$ au vu de la diversité des revenus des familles européennes et aussi parce que la ville est composée de quartiers européens et quartiers musulmans chacun ayant sa typologie de logements. Il serait erroné de dire que l'algérien découvre le logement européen en 1962 et que jusqu'à cette date il n'a connu que la maison traditionnelle, le bidonville ou l'habitat horizontal. L'expérimentation du logement européen par substitution s'enclenche dès 1954 et s'accélère entre 1960 et 1966 (Safar-Zitoun, 1996). Les familles musulmanes s'installent progressivement dans les logements des européens qui les quittent pour d'autres plus neufs et plus confortables. 1962 est aussi l'année de la prise de la ville (Sanson, 1974), et il est difficile de savoir combien de familles sont passées directement de la campagne, du bidonville, du camp de regroupement, du douar voire du village aux beaux quartiers des villes et à leurs logements libérés (MouazizBouchentouf, 2013). 


\section{Les actions des habitants sur le bâti : le décryptage des transformations}

8 C'est durant les années 1970 et essentiellement à travers les ZHUN, que s'enclenche en Algérie indépendante la construction en masse de logements collectifs. Le parc vacant en 1962 est considéré comme suffisant et fait reléguer cette question au second plan. Les manifestations de la crise de logements ne se font réellement sentir et à des degrés différents selon les villes qu'à la fin des années 1960. De tout l'héritage des périodes antérieures à l'indépendance, ce sont les logements construits durant le Plan de Constantine qui servent de modèle ${ }^{8}$ et qui sont repris : immeuble "boîte d'allumettes » et quadrillage des structures comme idéal de l'architecture, rejet des références architecturales et urbaines locales comme sources d'inspiration, primauté de la technique organisationnelle du chantier (chemin de grue, gestion méthodique du planning, surface de stockage) et de la rapidité de réalisation au détriment de la qualité architecturale et constructive.

Le modèle adopté de l'immeuble de logements collectifs construit en masse est aussi celui qui répond au mieux aux options politiques, idéologiques et symboliques du tout jeune état. Le gourbi (maison du paysan), la maison traditionnelle des médinas (Alger, Constantine, Mostaganem et Tlemcen) et la maison berbère (kabyle, mozabite ou des Chaouia) ou les ksour du sud sont rejetés car ils sont le symbole d'un archaïsme que le pouvoir en place veut gommer. Le même sort est réservé aux logements pour musulmans réalisés avant 1958 dont l'expérience est tout simplement occultée. L'objectif est de faire de l'algérien un homme moderne qui doit vivre dans un logement moderne. À l'ère du triomphe du fonctionnalisme et des préceptes de la Charte d'Athènes, et devant la vacance du domaine des études d'architecture et d'urbanisme ${ }^{9}$ important un savoir-faire et des conceptions de l'espace étrangères, c'est finalement le modèle des appartements ouverts sur l'espace public et superposés en barres essentiellement ${ }^{10}$ qui se généralise. Tout comme les technocrates du Plan de Constantine prétextaient l'urgence et l'économie pour faire imposer leur paradigme, les décideurs font de même pour les ZHUN : industrialisation de la construction, cellules-type, façades répétitives et monotones, plans de masses abolissant la rue et banalisant l'espace public, c'est ce qui caractérise les ZHUN qui ont marqué et qui marquent toujours le paysage urbain algérien.

L'inadaptabilité des logements collectifs au mode de vie de la famille algérienne est révélée dès les années 1970. La littérature, le cinéma, la presse, l'humour populaire mais aussi les travaux de chercheurs et praticiens dévoilent des conditions de vie difficiles, l'exigüité des logements et l'entassement de leurs habitants semblent en être les principales causes. Le logement européen, ce modèle standardisé est repris quasiment à l'identique jusqu'à aujourd'hui. Sa disposition a connu très peu de changements en dépit de toutes les transformations que lui « infligent ses occupants » (Lakjaa, 1998). Pourtant, le contexte socio-économique algérien est en grande mutation: diversité des modes de financement du logement ${ }^{11}$, abandon de la préfabrication dans le bâtiment et du système constructif du coffrage-tunnel à la fin des années 1980, mais aussi entrée en scène des bureaux d'études et des entreprises de la construction privées et des promoteurs immobiliers. Les décideurs et les concepteurs du logement social ont surtout l'héritage et l'expérience des grands-ensembles du Plan 
de Constantine et des ZHUN qui ont fait l'objet d'études et ont fait couler beaucoup d'encre. Cette "vulgate $»^{12}$ s'interroge sur l'appropriation des habitants de ce type de logements à travers les transformations/adaptations et le message qu'elles envoient.

11 L'éventail des actions sur le bâti s'élargit et se diversifie, tout comme les motifs de ces transformations qu'ils soient ouvertement dévoilés ou sous-entendus. L'origine rurale des familles avec des pratiques et des habitudes incompatibles avec le logement collectif a été une explication récurrente. Il est vrai que certaines activités comme travailler la laine, rouler le couscous ou laver et faire sécher les tapis ou le linge de maison qui se déroulaient traditionnellement dans la cour ne trouvent pas d'espace dans le logement en hauteur (Bendedouch, 1989). Il est également délicat de condamner un logement prévu pour cinq personnes qui en loge dans la réalité le double. Ce n'est pas tant le refus de la salle de bains ou de la cuisine à l'européenne (paillasse et évier obligeant à travailler debout alors que traditionnellement la femme prépare ses repas assise) qui explique leur transformation en chambre ou salon mais l'exigüité d'un logement devant recevoir une famille nombreuse ${ }^{13}$. C'est ainsi que les loggias ou balcons censés remplacer la cour de la maison traditionnelle sont annexés aux pièces qui leur sont contigües pour en augmenter la surface. Pourtant la cour est un espace polyfonctionnel, elle sert aussi bien au repas en plein air, à la lessive, à l'étendage du linge, au bricolage, à la sieste que pour les jeux des enfants (Deluz, 1988). Seulement le balcon ou la loggia vu leurs dimensions (leur surface dépasse rarement les $6 \mathrm{~m}^{2}$ ) se prêtent difficilement à ces activités. Si la salle de bains (le cabinet de toilettes est isolé) est supprimée et transformée en chambre c'est parce que le hammam (bain public) prend le relais de l'hygiène corporelle tout en étant un lieu de sociabilité (Semmoud, 2009). Enfin la cuisine peut aussi être sacrifiée et confinée dans la loggia ou le balcon.

12 De nos jours, cette amputation du logement de ces parties est une pratique presque inexistante. Ni salle de bain, ni cuisine n'ont été sacrifiées dans chacun des logements visités. L'évocation de cette possibilité a suscité un grand étonnement de la part des habitants pour qui il est impensable de vivre dans un logement sans ce confort minimum. La famille élargie vivant sous le même toit n'est plus une garantie de sa cohésion et de sa survie économique. La généralisation du salariat particulièrement chez la femme a précipité la décohabitation et fait baisser en même temps le taux d'occupation par logement ${ }^{14}$. Cette évolution de la famille algérienne et de son mode de vie se lit également à travers l'ameublement : la chambre des enfants avec lits, rideaux, draps et couettes imprimés de figures de dessins animés est devenue une priorité. Il n'est plus question de les faire dormir dans un séjour transformé la nuit en chambre comme ce fut le cas auparavant. Les photos de mariage du couple s'affichent sans gêne dans le séjour, tout comme sont accrochés aux murs de leur chambre les photos de vacances et les dessins faits par les enfants, une manière de marquer et de personnaliser l'espace. Enfin, l'importation massive de canapés et de meubles européens sonne le glas des sedariates ${ }^{15}$, "Ces banquettes surmontées de matelas et de coussins appariés à des accoudoirs, le tout recouvert d'un même tissu » (Lakjaa, 1997), qui ont l'avantage de servir aussi de lit.

13 Il est difficile de dire lequel des deux critères du déficit d'urbanité ou de la taille de la famille pèse le plus dans les transformations des logements tant les explications semblent dévoyées. Ces variables apparemment objectives (Safar-Zitoun, 2009) ne suffisent pas à rendre compte d'une réalité plus complexe, où le logement livré est 
« refusé » même par les plus nantis financièrement et culturellement, une catégorie considérée à priori comme maîtrisant le mode d'emploi du logement moderne. Au lieu de "nécessités et de besoins de la famille", d'autres raisons sont invoquées par les habitants comme la disponibilité des moyens financiers ou l'envie de mettre ce logement impersonnel au goût et au style de vie de la famille.

\section{Le « trois-pièces ${ }^{16}$ » : la persistance du type face à l'évolution de la famille}

14 Le «trois-pièces » objet de cette étude est le logement le plus fréquent dans la ZHUN Maraval et les 365 Logements Hai-Essabah. Ces deux ensembles de logements outre l'écart de vingt ans qui sépare leur réalisation présentent l'avantage de l'accès à la propriété de leurs locataires. Suite à la promulgation de la loi de cession des biens de l'état de 1981 reconduite à plusieurs reprises pour céder au fur et à mesure le parc locatif public, la plupart des familles sont propriétaires de leurs logements. Même si les habitants n'ont pas attendu d'être propriétaires pour agir sur le bâti, ce nouveau statut a fait accélérer les transformations. Le souci est de donner un aspect plus personnel au logement mais aussi d'augmenter sa valeur au cas où une opportunité de vente se présenterait.

15 Le logement de la ZHUN Maraval (figure 2a) est traversant (deux façades opposées), il regroupe les deux chambres face au séjour. La cuisine est proche des salles d'eau, le tout formant le bloc humide. Le séjour est divisé en deux pièces, l'intention était peutêtre d'offrir une salle à manger et un salon. Il est attenant à la porte d'entrée qui donne sur un dégagement, ce qui constitue la partie publique du logement. Le recours à la préfabrication (coffrage-tunnel) largement favorisée durant cette période d'industrialisation du bâtiment avec comme corollaire l'usage du béton emprisonne l'habitant dans la configuration imposée. Il est difficile d'abattre les murs intérieurs en béton sans fragiliser la structure du bâtiment, même si certains locataires l'ont fait sans grande conscience des dangers encourus particulièrement dans une zone sismique comme Oran (Semmoud, 2009). Le béton armé est également un matériau très dur contrairement à la brique creuse en terre cuite ou en plâtre plus facile à démolir.

La rigidité de ce trois-pièces ne permet pas de faire de grandes transformations qui se restreignent aux corps d'état secondaires: les revêtements de sol et des murs, la menuiserie, les équipements sanitaires (toilettes, lavabo, douche et évier) sont refaits. La seule transformation de taille consiste à intégrer les deux loggias de la cuisine et du séjour en déplaçant les menuiseries pour en augmenter la surface quitte à sacrifier ces espaces nécessaires pour la famille. Les entretiens avec les habitants ont fait ressortir leurs avis sur le réaménagement de leurs logements. Ils s'expriment au conditionnel comme s'ils avaient la possibilité de gommer quelques lignes sur un plan et de dessiner d'autres à leur place. Les principaux souhaits et vœux qui se dégagent sont :

- augmenter de quelques mètres-carrés la surface totale du logement, ce qui permettrait d'avoir quelques rangements inexistants dans le logement livré;

- déplacer la porte d'entrée des toilettes en l'orientant vers la cuisine plutôt que vers le séjour, une situation très désagréable particulièrement pour les odeurs qui s'en dégagent.

17 La disposition en $\mathrm{L}$ du séjour dégage une opinion partagée. Certains habitants estiment qu'il est contraignant d'avoir deux petites pièces déjà difficilement aménageables 
même quand elles sont réunies (la surface totale est de $19 \mathrm{~m}^{2}$ ). D'autres considèrent que c'est un avantage car cela permet d'avoir une chambre supplémentaire facilement séparable du séjour puisque chacun a sa propre fenêtre et de transformer ainsi le troispièces en un quatre pièces. Les habitants qui ont pour la plupart intégré les loggias soit à la cuisine soit au séjour auraient préféré avoir des balcons en saillie qui auraient servis pour étendre le linge et comme espaces de rangement.

Le trois-pièces de la ZHUN Maraval (1980):

Figure 2a : Le logement livré et les transformations réalisées ou souhaitées par les habitants

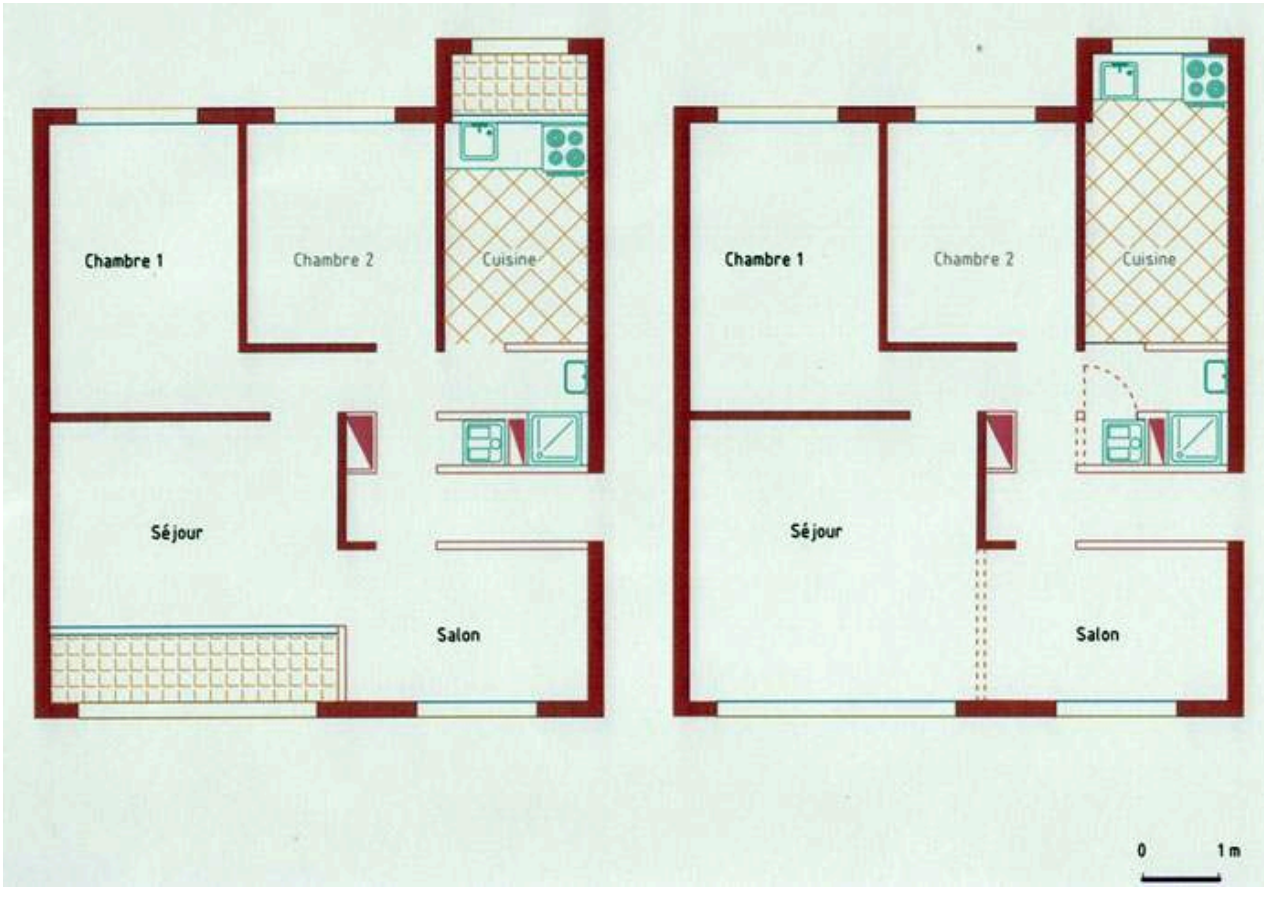

Figures $2 b$ et $2 c$ : Vues de l'extérieur

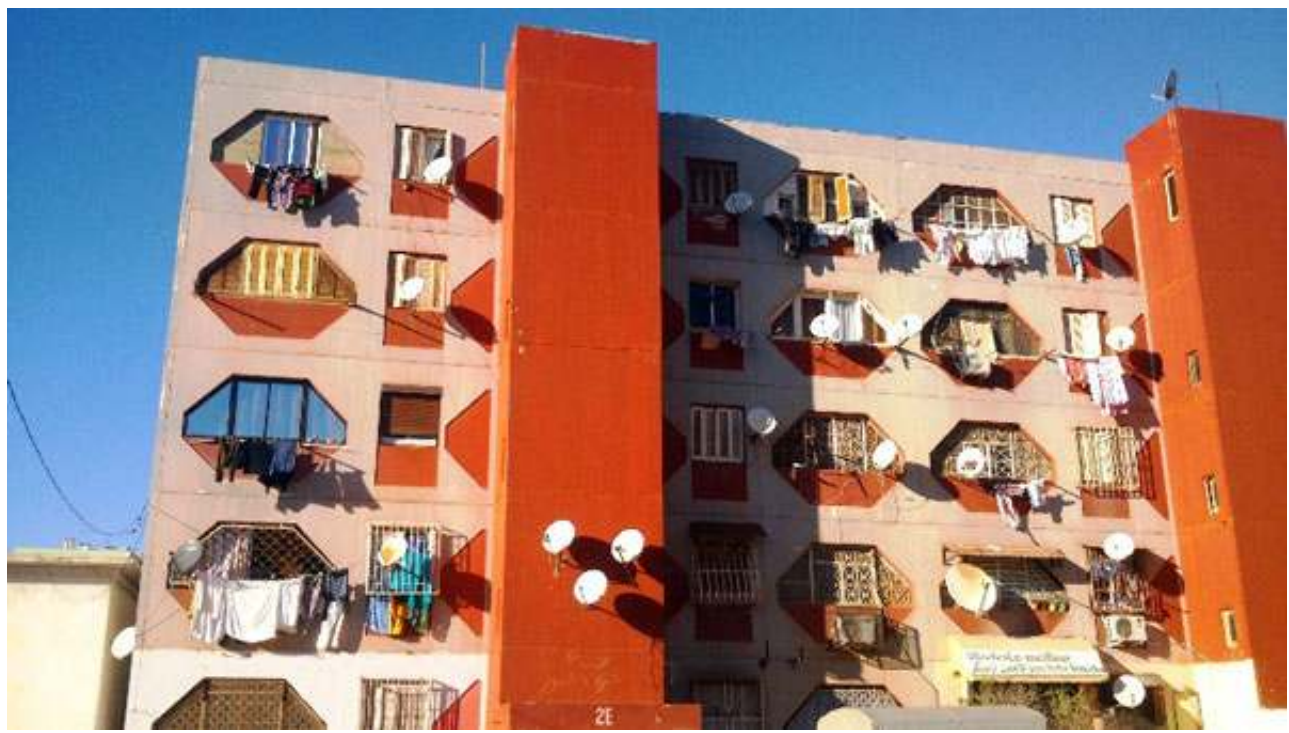




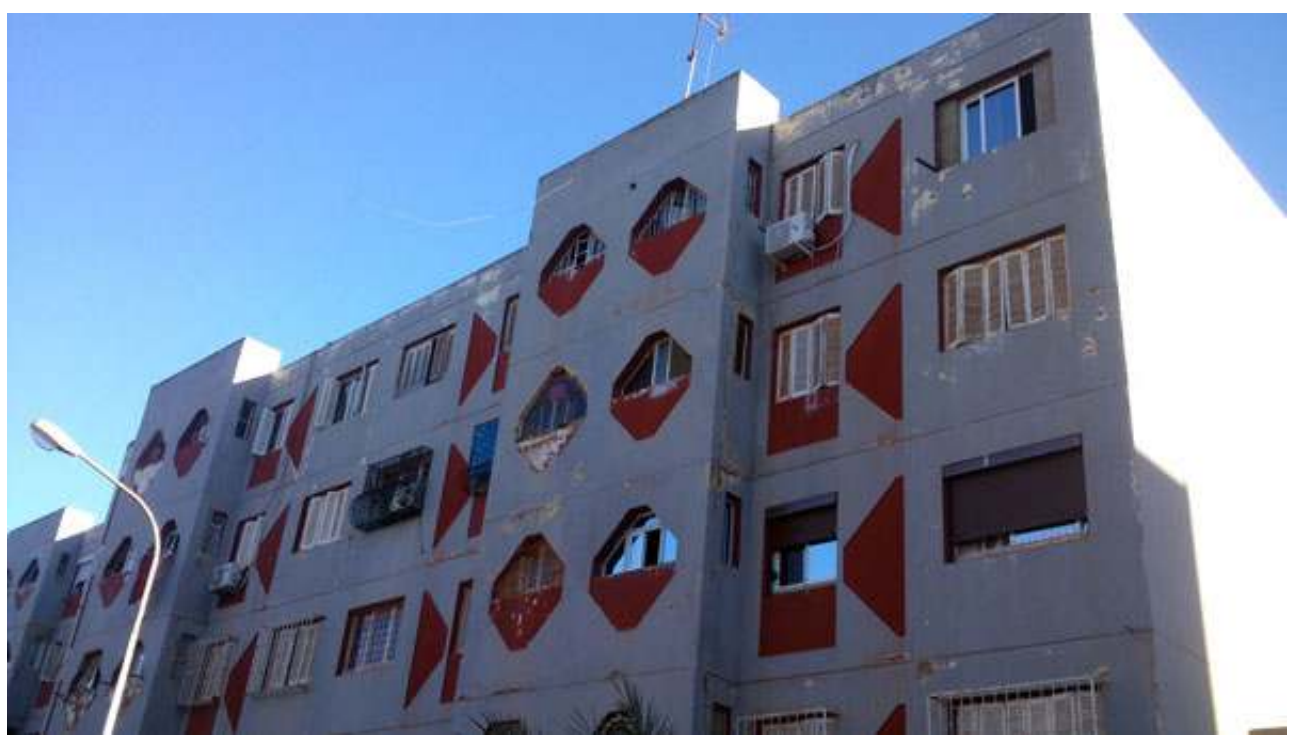

L'enquête aux 365 Logements Hai Essabah a ciblé les logements des enseignants universitaires qui représentent une partie des 365 logements de cette opération. Les entretiens avec les habitants ont fait également ressortir l'exiguïté des espaces, la réduction au maximum du balcon du séjour (aucune possibilité de l'intégrer à l'espace intérieur comme pour le logement de la ZHUN) et l'inconvénient d'une chambre commandée par le séjour (figure 3a). «Le logement est minuscule, il n'y a aucun placard, la cuisine est très petite, pas de terrasse côté séjour, la salle de bains est tellement petite qu'il n'y a qu'une douche et pas de baignoire ${ }^{17}$. La menuiserie, le carrelage, la peinture tout est de très mauvaise qualité. Lorsque je regarde les émissions de déco à la télé, les logements qui se font ailleurs qu'ils soient contemporains ou anciens, $j^{\prime}$ 'ai l'impression de vivre dans un couri ${ }^{18}$ 》 (un habitant du quartier Hai Essabah).

Cet entretien révèle une donne importante : l'ouverture de la famille aux télévisions étrangères et la généralisation de l'usage du web ${ }^{19}$ qui montrent des intérieurs de logements différents. L'agencement des pièces, l'ameublement, les tissus et les couleurs des murs que diffusent ces images sont considérés par les habitants comme la référence voire l'idéal à atteindre et devant lesquels le logement livré est très éloigné.

Les enquêtes et relevés ont fait ressortir deux types de transformations facilitées par l'indépendance des murs intérieurs de séparation des éléments porteurs (les poteaux) dans un contexte d'abandon de la technique du coffrage-tunnel. La première est la démolition du mur qui sépare le couloir du séjour afin d'augmenter la surface de ce dernier en supprimant la circulation et le dégagement de l'entrée. La seconde au contraire maintient le couloir et l'allonge d'un mètre pour changer la porte d'entrée de la chambre et éviter ainsi qu'elle ne soit commandée par le séjour. Ces deux types d'action sur le logement dénotent des choix de priorités dans une situation de rareté des mètres-carrés habitables. La première privilégie l'ouverture, le décloisonnement et l'exaltation de l'espace au détriment de l'intimité du séjour jugée secondaire. Pour la seconde, c'est la fermeture et l'indépendance de chaque espace (chambres et séjour) qui prennent le dessus quitte à augmenter la circulation au détriment de l'une des chambres qui perd un mètre-carré au profit du couloir. Il est vrai que la première est l'œuvre d'un enseignant-architecte plus sensible à la qualité des espaces, aux proportions et à la lumière mais rien ne dit que cet agencement ne fera pas d'émules

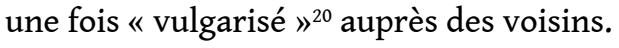


Le trois-pièces des 365 Logements Hai Essabah (2000) :

Figure 3a : Le logement livré, l'ouverture du séjour et le maintien de la circulation
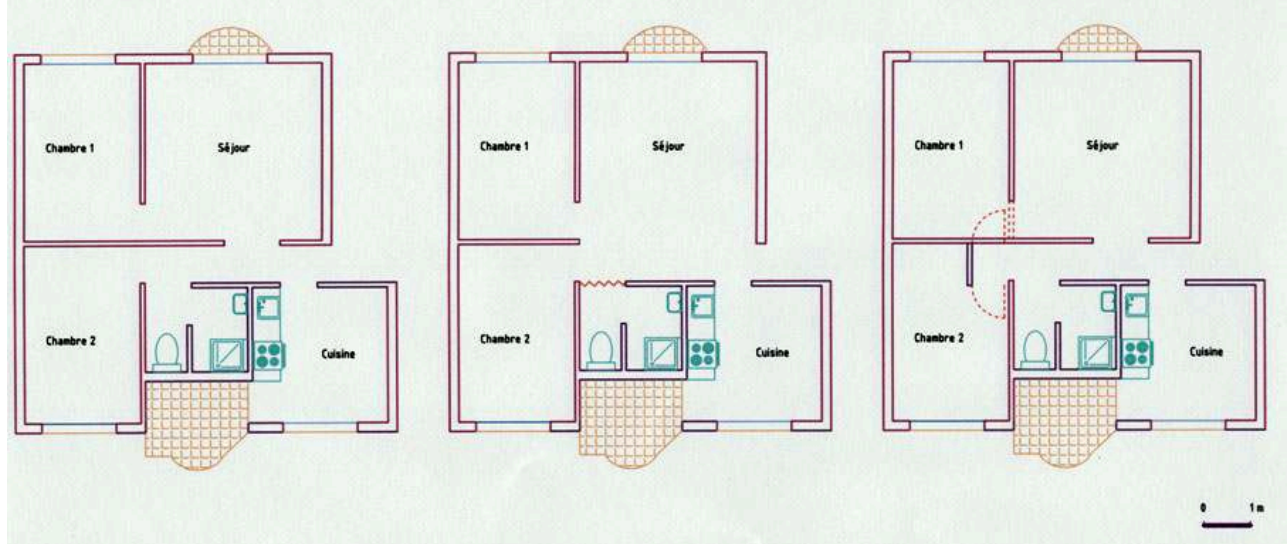

Figures 3b et 3c: Vues de l'extérieur

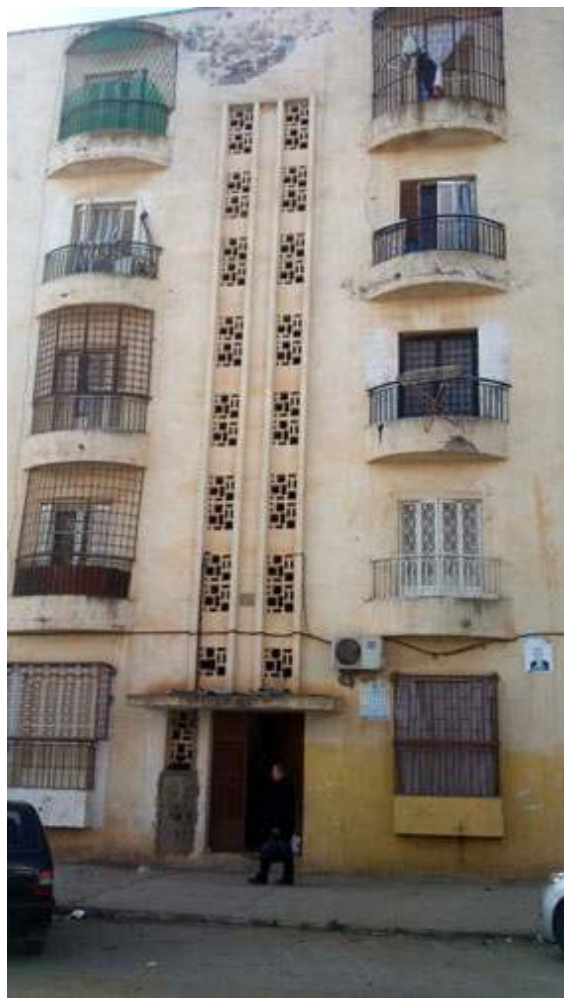




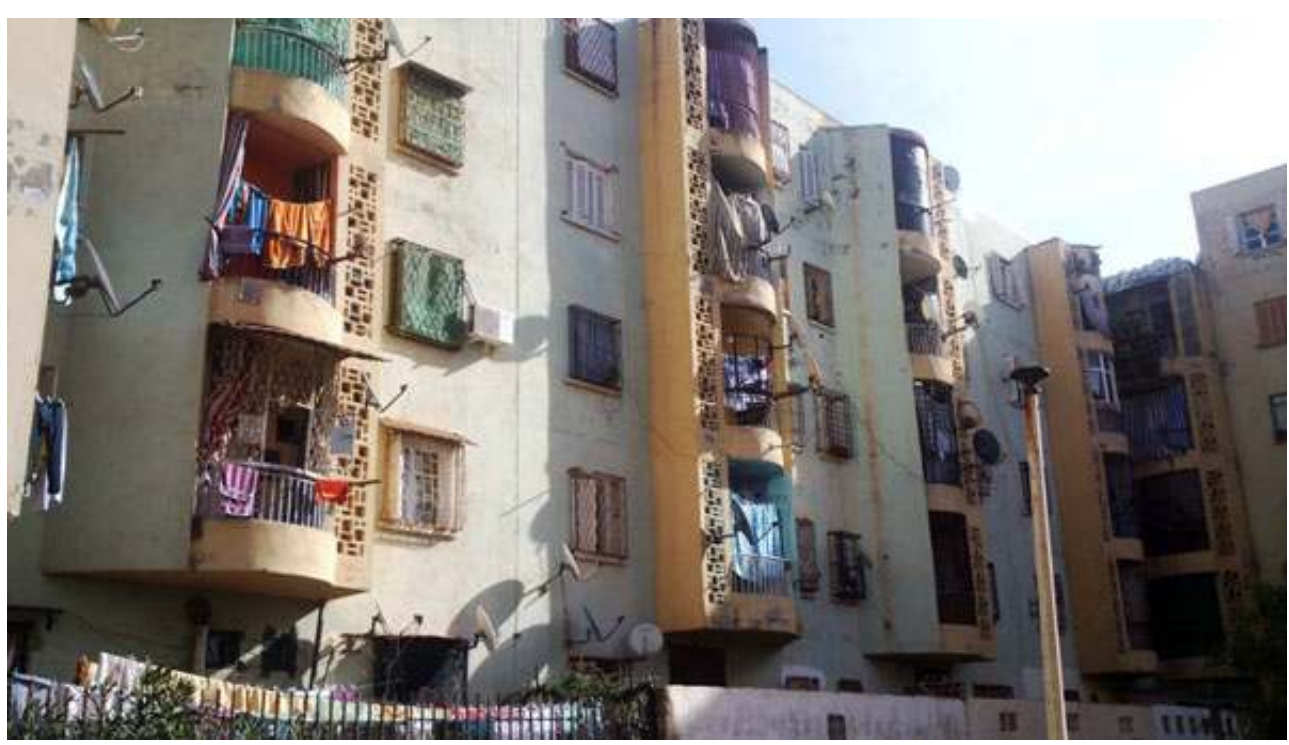

21 Le reste des travaux concerne l'embellissent du logement. À ce titre, les remarques des habitants sont très pertinentes : "Nous avons tout changé: le carrelage, la peinture, la faïence des salles de bain et de la cuisine, la menuiserie, quel gâchis! Ils auraient mieux fait de nous donner les logements nus, au moins nous n'aurions pas payé les démolitions et nous aurions peut-être eu un peu plus d'espace ». Les habitants sont donc conscients du plafonnement $\mathrm{du}$ coût $\mathrm{du}$ logement et suggèrent plus d'espace (les rangements et les terrasses notamment) et moins de prestations quitte à livrer un logement semi-fini qu'ils se chargeront d'achever chose qu'ils font de toutes les manières. Des propos à considérer avec des réserves car ils émanent d'une catégorie socioprofessionnelle élevée. Ce sont des familles qui à la remise des clés de leur logement ont pris le temps de le transformer avant d'y habiter, autrement dit qui étaient relativement à l'aise dans leur résidence antérieure. Ce logement social ne représente qu'une étape dans la concrétisation du projet résidentiel (Hadjij, 1998) : «Je vous confierai que je ne compte pas passer toute ma vie dans ce logement. Je l'ai pris juste comme dépannage, en plus je l'ai payé pour une bouchée de pain ${ }^{21}$. J'habitais chez mes beaux-parents et c'était l'occasion pour moi d'avoir mon chez-moi. Mon rêve est la maison individuelle, j'ai déjà acheté un terrain, actuellement je fais des démarches pour un prêt bancaire. Mais la maison n'est pas pour demain. C'est pour cela que j'ai fait des travaux, j'en ai au moins pour 10 ans ici, en plus il me rapportera plus quand je le vendrai » (entretien avec une habitante de Hai Essabah, mère de deux enfants).

\section{Le point de vue des concepteurs : réduction drastique de la marge de manœuvre de l'architecte}

"C'est le ministre qui dessine le logement, l'architecte ne fait qu'adapter"2 le plan de masse ", cette phrase est prononcée lors d'un entretien avec un architecte exerçant en privé pour le compte d'organismes publics de production de logements sociaux notamment l'OPGI. Confrontée au cahier ${ }^{23}$ des prescriptions techniques et fonctionnelles applicables aux logements sociaux (MHU, 2007), elle trouve tout son sens et résume bien la réalité affrontée par les concepteurs des logements sociaux. En effet, le logement est " encadré » par des chiffres, des seuils minima qui deviennent la règle, même les fenêtres et les portes-fenêtres ne sont pas épargnées. "Le tableau de 
menuiserie est très simple, il se résume au 1,20 m par 1,20 m ", une boutade que se lancent les architectes pour dénoncer le manque d'originalité des projets de logements sociaux.

Le logement est donc figé dans la disposition en partie jour et partie nuit, des chambres et un séjour obligatoirement commandés par un couloir, le tout ne dépassant pas $67 \mathrm{~m}^{2}$ pour un trois-pièce. Ces prescriptions le décrivent avec détails, rien n'est dit sur les autres logements, le trois-pièces étant le type le plus construit dans les programmes de logements sociaux et le seul depuis 2009. Au même moment, l'accent est mis sur la recherche de la qualité architecturale et urbaine tout en invitant les architectes à " Viser, comme objectif à obtenir une architecture aboutie, devant être perçue comme une réponse parfaitement concluante à une demande clairement dimensionnée et énoncée; Cette notion doit se traduire par l'adoption d'un agencement et d'une architecture qui dissuadent les occupants à procéder aux transformations de leur logement » (MHU, 2007). Les concepts de flexibilité et de " conception ouverte» du logement (Boudon, 1967) sont balayés et remplacés par l'exigence d'une connaissance parfaite de la demande de la part des architectes. Il est entendu par la demande non seulement la taille de la famille à laquelle est destiné le logement mais aussi son mode de vie, ce à quoi doit correspondre au maximum la réponse de l'architecte au point de ne susciter aucun rejet par les habitants.

De leur côté, les architectes de l'OPGI expriment leur malaise vis-à-vis des logements qu'ils font réaliser. Ils reconnaissent qu'ils n'en parlent qu'en chiffres et en résultats : « Ça fait vingt ans que je travaille à l'OPGI, j'en ai entendu des discours dans les réunions. Qualité, création, amélioration sont des mots qui reviennent souvent. Mais une fois les responsables dans leurs bureaux, d'autres mots émergent: combien de logements achevés, combien en cours, la quantité uniquement... Oui on aimerait bien faire mieux, mais avec le temps les injonctions $d u$ ministère sont de plus en plus sévères. Je dirais même que les logements des ZHUN étaient plus confortables. Aujourd'hui avec $67 \mathrm{~m} 2$, la salle de bain obligatoirement éclairée naturellement, 4 logements par étage, un programme des trois-pièces uniquement, un prix plafonné, un cahier des charges précis émanant d'Alger, que peut-on faire de mieux?».

À l'évocation de la possibilité d'augmenter les surfaces en laissant les locataires finir eux-mêmes leurs logements comme il est suggéré par quelques habitants interrogés, la réponse réside dans la difficulté justement à cerner cette famille qui va habiter ce logement: "Aussi bizarre que cela puisse paraître, le logement social est destiné à tout le monde, un programme achevé peut aussi bien être distribué aux cadres d'une entreprise, aux universitaires, à des bidonvillois dans le cadre du relogement ou je ne sais quoi encore. Nous ne savons jamais à qui il est destiné. Livrer un logement semi-fini ? Ça pourrait être une solution, mais sera-t-elle acceptée par tous?".

Réduire les surfaces au minimum vital, plafonner le coût dans un marché instable où les matériaux et la main d'œuvre sont en perpétuelle inflation et exiger en même temps la recherche, la connaissance et l'innovation dans la conception du logement, c'est museler toutes les bonnes intentions des concepteurs à améliorer le logement social.

\section{Conclusion}

27 Au terme des premiers résultats de cette étude, il est clair que le fossé qui sépare le logement social de ses habitants ne cesse de se creuser. Alors que l'Algérie est un pays majoritairement rural à la fin des années 1960 , sa population urbaine atteint $65 \%$ au recensement de 2006 bouleversant ainsi et les structures sociales et les modes de vie (Belguidoum, Mouaziz-Bouchentouf, 2010). Depuis l'indépendance, les familles de 
divers origines et revenus ont remodelé leurs logements pour l'adapter à leurs façons de vivre. Des pratiques elles aussi en constante évolution mêlant résidus de traditions et ouverture à l'occidentalisation dans une sorte de brouillage des références culturelles qui s'explique par le fait que peu d'algériens d'aujourd'hui ont vécu le logement en médina (Semmoud, 2009).

La méconnaissance ou l'occultation totale de la famille algérienne contemporaine ainsi que l'indifférence envers les travaux universitaires ou émanant de professionnels font que les acteurs ${ }^{24}$ de la production du logement restent cantonnés dans une logique d'urgence, d'échéances et de chiffres. De tous les modèles hérités c'est le plus efficace techniquement qui a été retenu. En cinquante années d'indépendance, aucune leçon n'a été tirée du " mauvais vieillissement » de ces ensembles de logements qui constituent la ville de l'Algérie indépendante.

Les recommandations des chercheurs (Belbacha-Merouche, 2009 ; Tebib, 2008) insistent sur le rôle des habitants et la nécessité de les impliquer en amont du processus de production, autrement dit au moment de la conception du logement particulièrement pour les nouvelles formules de financement. Elles avancent également comme nécessaire et urgente l'augmentation de la surface des logements, ce qui rejoint les souhaits des habitants mais semble un vœu pieux au vu du plafonnement des coûts. Les architectes en tirant la leçon du «logement habité » transformé par ses habitants envers lequel ils ne peuvent rester éternellement indifférents se doivent de reconsidérer le modèle conçu du logement social. Faut-il livrer un logement semi-fini mais plus spacieux comme le suggèrent les habitants? Ce serait une piste à explorer, une solution à expérimenter. Rien n'est possible sans une réelle volonté politique ${ }^{25}$ et sans l'ouverture d'un débat quasi inexistant aujourd'hui en Algérie sur la question du logement social de demain.

\section{BIBLIOGRAPHY}

Arrif A., 2000, «Compétences habitantes : plans en projet, plans en acte. Quelques éléments de réflexion », in Berry-Chikhaoui I., Deboulet A., Les compétences des citadins dans le monde arabe : Penser, transformer la ville, Paris, Tunis, Tours, IRMCKarthala- Urbama, p.51-64.

Almi S., 2002, Urbanisme et colonisation, présence française en Algérie, Hayen, Mardaga, 155 p.

Bekkar R., Sfaxi S., 2000, «Reformuler l'espace : compétences et savoir-faire des habitants à Bizertz (Tunisie), in Berry-Chikhaoui I., Deboulet A., Les compétences des citadins dans le monde arabe : Penser, transformer la ville, Paris, Tunis, Tours, IRMCKarthala- Urbama, p.63-77.

Belbacha-Merouche C., 2009, Le logement social participatif. Pour une approche participative dans la conception. Cas de la ville nouvelle Ali Mendjli, mémoire de magistère en architecture, Université de Constantine, $50 \mathrm{p}$.

Belguidoum S., Mouaziz-Bouchentouf N., 2010, «L'urbain informel et les paradoxes de la ville algérienne : pratiques urbaines et légitimités sociales », Espaces et sociétés, N 143, p.101-116. 
Belguidoum S., Millet D., 1985, « Détournements et retournements des modèles urbains et architecturaux à Sétif », in Haumont N., Marie A., Politiques et pratiques urbaines dans les pays en voie de développement, Tome 2, Paris, L'Harmattan, p.228-247.

Bendedouch A., 1989, Les nouvelles zones d'habitat collectif dans les grandes villes, cas d'Oran, mémoire de magistère en urbanisme, Université des Sciences et de la Technologie d'Oran 276p.

Boudon P., 1977, Pessac de Le Corbusier. 1927-1967,étude socio-architecturale, Paris, Bordas, 153 p.

Celik Z., 1997, Urban Forms and Colonial Confrontations. Algiers under French Rules, Los Angeles, University of California Press, 196 p.

Davidovitch A., 1968, « Raymond H., Haumont N., Raymond M.-G., Haumont A., L'habitat pavillonnaire. Haumont N., Les pavillonnaires. Etude psycho-sociologique d'un mode d'habitat. Raymond M.-G., La politique pavillonnaire ", Revue française de sociologie, 9-2. pp. 269-274.

Deluz J.-J., 2010, Le tout et le fragment,Alger, Barzakh, 381 p.

Deluz .J-J., 1988, L'urbanisme et l'architecture d'Alger. Aperçu critique, Alger, OPU, Liège, Mardaga,197 p.

Hadjij C., 1998, « Famille, logement, propriété à Alger », Insaniyat, n 4, p.99-107.

Lakjaa A., 1998, « La ville : creuset d'une culture nouvelle. (Villes, cultures et société en Algérie) », Insaniyat, $\mathrm{n}^{\circ} 5, \mathrm{p} .39-59$.

Lakjaa A., 1997, «L'habiter identitaire : éléments pour une problématique d'une urbanité en émergence », Insaniyat, $n^{\circ} 2$, p.77-103.

Le Corbusier, 1935, La ville radieuse, Paris, Vincent et Fréal, 345 p.

Léger J.-M., Decup-Pannier B., 2005, « La famille et l'architecte : les coups de dés des concepteurs ", Espaces et sociétés, 2005/2 n 120-121, p.15-44.

Meyer R., 1959, « L'habitat social en Algérie dans le cadre du plan de Constantine », Techniques et architecture, $20^{\mathrm{e}}$ série $-\mathrm{n}^{\circ} 1, \mathrm{p} .8-10$.

MHU, 2007, Prescriptions techniques et fonctionnelles applicables aux logements sociaux, ministère de l'habitat et de l'urbanisme, $52 \mathrm{p}$.

Mouaziz-Bouchentouf N., 2013, Foncier et immobilier à Oran. Législations et stratégies d'acteurs, thèse de doctorat en urbanisme, Université des Sciences et de la Technologie d'Oran, $380 \mathrm{p}$.

Picard A., 1994, « Architecture et urbanisme en Algérie. D'une rive à l'autre (1830-1962) », Revue du monde musulman et de la Méditerranée, $\mathrm{n}^{\circ} 73-74, \mathrm{p} .121-136$.

Pinson D., 1994, « Maroc : un habitat "occidentalisé" subverti par "la tradition" », Monde arabe Maghreb-Machrek, $n^{\circ} 143$, «Villes dans le monde arabe », La Documentation française, premier trimestre 1994, p.190-203.

Safar-Zitoun M., 2009, « Digressions sur l'"Algérois" », Les Cahiers d'EMAM, n 18, p.21-28.

Safar-Zitoun M., 1996, Stratégies patrimoniales et urbanisation : Alger 1962-1992, Paris, l'Harmattan, $297 \mathrm{p}$.

Said A., 2005, « Le logement social urbain et la dynamique spatiale. Stratégie des acteurs décideurs à Oran (1990-2000) », Insaniyat, n²9-30, p.231-247.

Sanson, H., 1974, «Prise de la ville, prise du pouvoir », in Villes et sociétés au Maghreb. Etudes sur l'urbanisation, Paris, Editions du Centre National de la Recherche Scientifique, p21-28. 
Semmoud B., 2009, « Appropriations et usages des espaces urbains en Algérie du Nord », Cahiers de géographie du Québec, vol. 53, n 148, p.101-118.

Tebib E., 2008, L'habiter dans le logement de type social à Constantine. Manières et stratégies d'appropriation de l'espace, thèse de doctorat en urbanisme, Université de Constantine, 387 p.

Tric O., 1999, Conception et projet en architecture, Paris,L'Harmattan, 314 p.

\section{NOTES}

1. Par maître d'ouvrage, il est entendu toute personne physique ou morale qui se charge de financer et de diriger une opération de réalisation de logements sociaux.

2. Les deux auteurs qui ont réalisé une partie de leurs enquêtes pour le Plan Construction relèvent durant les années 1970 un changement d'attitude des architectes plus attentifs à une demande diversifiée contrairement à leurs ainés qui déclaraient se méfier de la parole des habitants.

3. Réalisation Expérimentale, ce classement garantit une aide financière du ministère de l'Équipement et du Logement et un suivi sociologique de l'opération jusqu'à l'appropriation par les habitants (Tric, 1999).

4. Zone d'Habitat Urbain Nouvelle, une procédure mise en place en 1975 permettant de réaliser les logements sociaux ainsi que les différents réseaux et équipements d'accompagnement et abandonné à la fin des années 1980.

5. Dans sa préface de l'habitat pavillonnaire (1966), Henri Lefebvre précise qu'une enquête sur le logement et avec les habitants ne peut se faire par le biais du questionnaire qui est la technique classique des sociologues mais avec l'entretien non directif complété par la description (Davidovitch, 1968).

6. Parmi elles, il y a le projet théorique de cité indigène de Louis Bérthy (1936), l'esquisse initiale de la cité Scala au Clos Salembier à Alger (Seiller, Lathuillière, 1935) et le projet d'aménagement de la cité musulmane à Annaba ex-Bône (Danger, 1931).

7. En type, en taille et en standing.

8. Avec toutefois une nette amélioration des surfaces et du confort.

9. En 1962, l'Algérie ne compte pratiquement qu'un seul architecte « algérien » (Deluz, 1988).

10. La tour, du fait de l'usage obligatoire de l'ascenseur, est très rare.

11. Alors que le logement social est entièrement financé par l'état, d'autres formules sont introduites dès 1990 impliquant une participation financière du postulant au logement avec une aide réduite de l'état.

12. Il s'agit des mémoires de licence ou de magistère en architecture, en sociologie ou en géographie urbaines, ainsi que les thèses de doctorat.

13. La sur-occupation du logement est due soit à la cohabitation des ménages soit au nombre élevé d'enfants dépassant les capacités d'accueil du logement.

14. Selon l'office national de statistiques ce taux ne cesse de diminuer passant de 7,82 personnes par logement en 1977, à 6,70 en 1987 et à 4,89 en 2009.

15. Le mot arabe "seddari» (pluriel «seddariate») est la banquette sur laquelle on peut s'asseoir ou dormir (Lakjaa, 1997).

16. En Algérie, les chambres et le séjour sont considérées comme pièce. La cuisine, les toilettes et la salle de bains sont sous-entendus et non mentionnés.

17. On ne peut s'empêcher de trouver en écho à ces propos ceux de Jean Nouvel « un bon logement est un logement grand» une forte intention qu'il expérimente dans les projets de logements à Saint-Ouen, Nîmes, Bezons et Tours (Tric, 1999 ; Léger, Decup-Pannier, 2005).

18. Déformation du mot écurie qui veut dire un lieu tout juste bon pour abriter des animaux. 
19. Soit à domicile soit à travers les cybercafés qui offrent une connexion à 60 dinars l'heure (0,40 euros en 2013).

20. La découverte du même logement aménagé différemment peut donner des idées à lesquelles on n'avait pas pensé soi-même.

21. Ces logements sont cédés par l'OPGI (Office de Promotion et de Gestion Immobilière) à 600000 dinars algériens soit 4000 euros en 2013. Leur revente pourrait rapporter dix fois plus.

22. Beaucoup d'architectes déclarent subir le diktat des maitres d'ouvrage publics qui négocient les honoraires proposés par les bureaux d'études sous prétexte que les logements et les assemblages sont toujours les mêmes, seul le plan de masse varie avec le contexte et la situation du terrain.

23. Ce n'est pas un règlement mais des indications relatives au logement et à l'ensemble (forme urbaine, notion de quartier...) mais qui sont devenus une sorte de bible de tous les maîtres d'ouvrage chargés de la réalisation de logements sociaux.

24. Le ministère, les organismes publics, les maîtres d'œuvre et les maîtres d'ouvrage, les entreprises, etc.

25. Fernand Pouillon aurait-il réalisé ses ensembles de logements à Alger sans la volonté du maire Jacques Chevallier?

\section{ABSTRACTS}

The Algeria since independence has provided a lot of effort in social housing. Housing designed and built by practitioners and reshaped by their inhabitants to suit their lifestyles. Researchers from various disciplines decipher these changes and uncover their meaning while professionals reproduce the same forms and arrangements in a kind of autism to scientific production and to the evolution of the Algerian family. This article by interrogating the practices inhabitants tries to weave a link between the conceived and constructed flat and the flat wished by the inhabitants and revealed by the research.

Depuis son indépendance, l'Algérie a fourni beaucoup d'efforts en matière de logement social. Des logements dessinés et construits par les praticiens puis remodelés par leurs habitants pour les adapter à leurs modes de vie. Les chercheurs de diverses disciplines décryptent ces modifications et mettent au jour leurs sens alors que les professionnels reproduisent les mêmes formes et dispositions dans une sorte d'autisme envers la production scientifique d'une part et l'évolution de la famille algérienne d'autre part. Cet article, en interrogeant les pratiques habitantes, tente de tisser un lien entre le logement conçu et construit et le logement désiré par les habitants et révélé par la recherche.

Seit Erreichung der Unabhängigkeit hat sich der algerische Staat im Bereich des sozialen Wohnbaus stark engagiert. Ergebnis waren und sind Wohnungen, die von Fachleuten entworfen und gebaut, danach aber von den Bewohnern umgestaltet werden, um sie ihren Lebensbedürfnissen anzupassen. Forscher verschiedener Wissensdisziplinen versuchen, diesen Anpassungen zu untersuchen und ihren Sinn einer Allgemeinheit bewusst zu machen, doch die Verantwortlichen und Ausübenden des Wohnbaus reproduzieren immer wieder dieselben baulichen Formen und Anordnungen, als ob sie taub und blind gegenüber der Forschung und dem Strukturwandel der algerischen Familien wären. Dieser Artikel untersucht die Wohnpraxis 
um eine Beziehung zwischen verschiedene Aspekte der Wohnung herzustellen, nämlich die gebaute und gelebte Wohnung und auch die Wohnung als Fachinteresse.

\section{INDEX}

Mots-clés: habitants, logement conçu, logement social, logement vécu, Oran, transformations du logement

Keywords: housing designed, housing experience, Oran, residents, social housing, transformations of housing

Schlüsselwörter: Bewohner, Oran, sozialer Wohnbau, Wohnungentwurf, Wohnungserleben, Wohnungsumgestaltung

\section{AUTHOR}

\section{NAJET MOUAZIZ-BOUCHENTOUF}

Architecte, maitre de conférences, département d'architecture de l'Université des Sciences et de la Technologie d'Oran Mohamed Boudiaf - El Mnaouar, BP 1505, Bir El Djir 31000 Oran najetmouaziz@yahoo.fr 